\title{
Piston Pad Device
}

National Cancer Institute

\section{Source}

National Cancer Institute. Piston Pad Device. NCI Thesaurus. Code C50104.

A pad designed to fit onto the head of a piston. 